\title{
Twitter Issue Response Hashtags as Affordances for Momentary Connectedness
}

Social Media + Society July-September 2018: I-14 (C) The Author(s) 2018 Reprints and permissions: sagepub.co.uk/journalsPermissions.nav DOI: 10.1।77/2056305। I8784780 journals.sagepub.com/home/sms (S)AGE

\author{
Chamil Rathnayake' (D) and Daniel D. Suthers ${ }^{2}$
}

\begin{abstract}
Framing online collective behavior within theoretical frameworks developed to describe offline phenomena is common in social media studies. This can limit the scope of analysis because the interplay between user capabilities, intentions, and platform architecture makes online exchanges different from offline interaction. We introduce a novel conception of online publicness, momentary connectedness, which is a structure of polymorphic computer-mediated sociality that includes transactive as well as non-transactive phenomenological online activity. We suggest that Twitter issue-response hashtags can be seen as affordances for momentary connectedness where Tweets that display broad and vague audience dimensions, as well as clusters with different interactional orientations, co-exist within the same topical network. We illustrate this polymorphism of momentary connectedness with a case study of an issue-response network.
\end{abstract}

\section{Keywords}

social media, publics, communities, momentary connectedness

\section{Introduction}

Scholars who examine collective behavior on social media commonly frame online collective activity within theoretical perspectives developed to describe offline phenomena, such as communities (e.g., Albrechtslund, 2010; Velasquez, 2012) and publics (e.g., Maireder \& Schlögl, 2014; Shklovski \& Valtysson, 2012; Tremayne, Zheng, Lee, \& Jeong, 2006). While this approach has its merits in terms of its ability to provide a theoretical foundation for a relatively new field of study, its potential of describing online phenomena is limited due to differences between online and offline spaces. Despite the fact that several scholars (e.g., Fernback, 2007; Kruckeberg \& Vujnovic, 2010; Mejias, 2010; Yuan, 2012) problematize the use of offline metaphors to theorize online spaces, many social media studies have been built on the basis that new media platforms resemble offline collective phenomena. This can limit the scope of analysis, as the online activity results from the interplay between user capabilities and platform architecture, which makes online exchanges different from offline interaction. As the shift from industrial to post-industrial societies resulted in novel conceptualizations, the rise of networked societies also demands new conceptualizations. In particular, this need emerges because, as boyd (2011) noted, networked technologies offer affordances - the interplay between technology and user that offer possibilities for or constrain action (Evans, Pearce, Vitak, \& Treem, 2017)—that can bring about new dynamics of participation.

There is a growing body of literature that conceptualizes new media platforms by considering their architecture, affordances, and novel ways in which technical elements restructure communication among different groups of users. In general, this scholarly discourse consists of studies representing two branches of the topic: (1) work that discusses how platform architecture shapes interaction and collective behavior (e.g., boyd, 2011; Bruns \& Burgess, 2011; Bruns \& Moe, 2014) and (2) studies that focus on novel conceptions of online interaction, such as connected presence (Licoppe \& Smoreda, 2005), public sphericules (Holmes, 2002), and digital togetherness (Marino, 2015). Although these studies capture nuanced aspects of online activity, the field requires more work that conceptualizes the use of specific social media platforms. This need arises due to at least two reasons.

\footnotetext{
'University of Strathclyde, UK

${ }^{2}$ University of Hawaii, USA

Corresponding Author:

Chamil Rathnayake, University of Strathclyde, Glasgow G4 OLT, UK.

Email: chamil.rathnayake@strath.ac.uk
} 
First, digitally native conceptions of online collective behavior are needed to address the issue of over-dependence on offline metaphors, instead characterizing new dynamics arising from use of the unique affordances of such media. Second, networked technologies offer an expanded sociality that allows co-occurrence of multiple forms of collective behavior as well as quasi-public forms of communication, yet there is a dearth of work that examines the polymorphic forms of collectivity in these spaces. In this article, we introduce momentary connectedness (MC), a novel conception of online publicness, as an extended computer-mediated sociality that includes transactive as well as non-transactive online activity. We argue that this sociality is polymorphic and structural as well as phenomenological, as it is a broader space in which some utterances form transactive structures while the others may not be projected for a specific audience. We focus on issue-response networks (IRN) - networks defined by hashtags created in response to an issue or eventto develop the notion of MC, and use the Twitter hashtag \#NepalEarthquake as a case to demonstrate polymorphism (i.e., different forms of collective phenomena) in IRNs.

Twitter use has been subject to substantial investigation from a broad range of perspectives. The vast majority of Twitter studies explore how the tool is used for various purposes, such as politics (e.g., Himelboim, Hansen, \& Bowser, 2013), cultural conversation (e.g., Brock, 2012), and cultural performance (Florini, 2014). The number of studies that conceptualize the act of tweeting itself remains insufficient. Studies conducted by several researchers provide a starting point to conceptualize Twitter use beyond offline theoretical foundations. For instance, Licoppe and Smoreda's (2005) notion of connected presence describes how communication technologies have enabled interstitial or phatic communications that support quasi-continuous forms of togetherness at a distance. Moreover, several studies help argue that such quasi-continuous aspects of communication can include phenomenological elements. For instance, Litt's (2012) work showed how Twitter use is characterized by users' imagination of their audiences. Moreover, Marwick and boyd's (2011) study showed that tweeting can involve public rejection of audience, self-recording of the lives of senders, and being uncomfortable in identifying interlocutors, as well as strategic use of the platform, such as creation and marketing of personal brands. Gruzd, Wellman, and Takhteyev (2011) also argued that Twitter networks are both real and imagined.

We use the concept of uptake - "acts in which one participant takes up another's contribution and does something further with it" (Suthers, 2006, p. 331) - to situate MC in a digitally native context. Uptake is the "most fundamental element of interaction" (Suthers, Dwyer, Medina, \& Vatrapu, 2010). This concept provides a basis for analyzing interaction independent of media, so is not subject to the constraints that offline metaphors carry over to the analysis of online transactions, and therefore provides a foundation for a natively digital conception of phenomenological elements of online expressions. In this article, we introduce a related concept, projected uptake, which is based on the affordances of acts for future uptake. We use these two concepts to examine transactive as well as non-transactive elements in Twitter hashtags. Hashtags are affordances of the platform that organize instances of MC into networks. We discuss these instances at levels of mediated communication, including original tweets, utterances that take up expressions of others (retweets and quote tweets), and expressions that can initiate interactional exchange (replies and mentions).

This article is structured as follows. First, we discuss previous work that examines publicness on social media. In the next section, we highlight the need for natively digital conceptions of online publicness. This is followed by a discussion of uptake and projected uptake, building a basis for a natively digital account of online sociality. Then, we use a sample of the \#NepalEarthquake Twitter network as an IRN to empirically examine polymorphism.

\section{Publicness on Social Media}

The need to problematize current conceptualizations of publicness on Twitter arises from a dearth of theoretical perspectives that move beyond frames provided by the study of offline behavior. Current new media literature includes a substantial number of studies that draw on three offline phenomena to describe online interaction: (1) public sphere, (2) publics, and (3) communities. In particular, a substantial number of studies that examine these three notions in online settings emerged from the same scholarly community. In this section, we examine these theoretical lenses to identify their ability or limitations in describing nuances in social media platforms, and stress the need for novel conceptualizations.

\section{Public Sphere, Issue Publics, and Communities}

The body of social media literature consists of a substantial number of studies that view online platforms as spaces that resemble the notion of public sphere (Ausserhofer \& Maireder, 2013; Bruns, Burgess, Highfield, Kirchhoff, \& Nicolai, 2011; El-Nawawy \& Khamis, 2010). Naming domains of online activity as "spheres," such as "blogosphere" (Al-Ani, Mark, Chung, \& Jones, 2012; Tremayne et al., 2006), and "Twittersphere" (Ausserhofer \& Maireder, 2013), is common in social media literature. Public sphere, coined by Jürgen Habermas, is a "constellation of communicative spaces in society that permit the circulation of information, ideas, debates - ideally in an unfettered manner - and also the formation of political will" (Dahlgren, 2005, p. 148). Dahlgren discusses three dimensions of the public sphere: (1) structures/institutional features (e.g., media organizations), (2) representation/media outputs (e.g., "massification" of communication, and (3) discursive interactional 
processes. Accordingly, the term public sphere connotes a fluid space characterized by a focus on classic democratic issues (e.g., freedom of speech, inclusion/exclusion), and questioning of accuracy, fairness, completeness, ideological tendencies, and agenda setting through media outputs, as well as discursive practices. In particular, the public sphere has been conceived of as a collective space for rational argumentation and opinion formation.

Although networked technologies seemed to appear (especially during the early stages of the social web) as promising spaces where the public sphere can be sustained, scholarly work on the topic indicates mixed views on such potential. For instance, Dahlgren (2005) views the potential of the Internet to destabilize political communication systems as a positive impact that can help disperse old patterns. Dahlgren also notes that the Internet can allow citizen engagement in counter publics that can lead to new democratic practices. Geiger (2009) suggests that the question: "what is the role of the Internet-based discourse communities in the constitution of the public sphere?" is more appropriate than "is the Internet (part of) a new public sphere?" (p. 24). Highlighting the role algorithms play in the new space, Geiger notes that the blogo/public sphere operates via Habermasian discourse on a microlevel, while the macrolevel structure is constructed by algorithms, which in turn can be more powerful, invisible, and uncontestable. While Dahlgren (2005) and Geiger (2009) view the Internet as a positive development, some scholars show that networked technologies may have mixed effects on the formation and performance of the public sphere. For instance, Papacharissi (2002) argues that although communication technologies can enrich political discussion by providing access to information, the Internet can cause fragmentation of political discourse and compromise representativeness. Rauchfleisch and Kovic (2016) suggest a hierarchical model that shows that the Internet can make a high impact in agenda-setting and a low impact in deliberation. Their model also shows that the Internet can exert only a medium-level impact on identity building, and control and criticism.

Although some scholars see electronic networks enhancing the performance of the public sphere (e.g., Boeder, 2005; Dahlgren, 2005), this does not mean that the Internet will eventually transform into a public sphere. In general, studies discussed above view the Internet as a primarily interactive space that may help perform a phenomenon that emerged (or was conceptualized) offline. However, this view is only partly true as modern new media platforms allow multiple forms of expressions that include but are not limited to transactive exchanges. For instance, it is possible that Twitter users take up an affordance (e.g., a hashtag) for expressive purposes, rather than to reach a specific audience. Moreover, online expressions and transactions are not limited to political conversations, debates, and deliberation. In particular, this can be the case with respect to IRNs as they emerge momentarily in response to issues or events.

\section{Publics and Issue Publics}

Researchers have explained the interactive space enabled by the Internet, envisioning it as a domain for "publics" redefined by the logics of networked technologies. For example, examining online engagement in oppressive contexts, Shklovski and Valtysson (2012) argue that various types of publics (e.g., issue, mundane, and counter publics) can foster social change online even in authoritarian contexts. The most common conception of publics is characterized by a common interest and a sense of being part of a collective. For instance, John Dewey's and Herbert Blumer's work that laid the foundation for the concept of publics show that this concept can be characterized by a group of individuals who face a common issue, recognize it and its effects, and organize to address it (Frederick \& Neuwirth, 2008). However, the discourse perspective suggested by Warner (2002) shows that the common interest is a projection of a discourse itself rather than an external property. Warner argues that a public is a discursive space organized by the discourse itself that can be characterized by an orientation to reach strangers, personal and impersonal public speech, dependence on attention given by the members, reflexive circulation of discourse, temporality in circulation, and poetic world making. Warner's conception of publics suggests that while texts themselves do not create publics, the concatenation of texts through time gives rise to a public. He notes that "only when a previously existing discourse can be supposed, and when a responding discourse can be postulated, can a text address a public" (p. 420 ). Warner also argues that a public is enacted with the attention given by the members and ceases to exist in the absence of attention. This perspective is more appropriate to understand the extent to which the notion of publics can help describe online issue responses.

Online issue responses may not necessarily be discursive for several reasons. First, as mentioned previously, nontransactive expressions are common on the Internet as there can be instances in which the users may merely post expressively without engaging in an active discourse. Second, the multiplicity of information or posts that appear on a user's Twitter feed or Facebook stream may limit the attention that he or she pays to each issue/topic. In other words, text representing multiple discourses that appear simultaneously on one's social media feed may affect user attention on given discourse and limit reflexive circulation. Attention is the main category used to differentiate between members and non-members of a public (Warner, 2002). Simultaneous exposure to text representing many discourses may make engagement platform-centric rather than issue/topic-centric. Platform-centric engagement with a multitude of topics/ issues may shift the sense of being collective from the discourse to the platform. Furthermore, the ranking algorithms that personalize social media feeds may affect the extent of exposure to a given discourse even among those who use the same social media platform. Due to these reasons, the notion 
of publics may not necessarily provide a clear perspective on the dynamic nature of social media.

Some scholars have captured nuances of computer-mediated communication in their definitions of online publics. For instance, boyd (2011, p. 39) defines networked publics as "publics that are restructured by networked technologies" that are "simultaneously (1) the space constructed through networked technologies and (2) the imagined collective that emerges as a result of the intersection of people, technology, and practice." She further notes that new possibilities for interaction can lead to new dynamics that shape participation. Arguably, it is this very set of new possibilities that problematize the notion of publics itself when applied to online contexts. Specifically, the fluid nature of online communication blurs the boundary between the private and the public and allows for interactive, quasi-interactive, as well as non-interactive expressions simultaneously, causing a fragmentation of the online space and weakening our confidence in identifying clear "networked publics."

The notion of issue publics has been used to frame social media activity related to relatively specific domains, such as issue responses (e.g., Bruns \& Burgess, 2011; Bruns, Moon, Paul, \& Münch, 2016). Tremayne et al. (2006) "cast” the network of Iraqi war blogs as an "issue public," a group comprising highly educated, professional, male bloggers that does not resemble the general population. Maireder and Schlögl (2014) identify the hashtag "\#aufschrei” as an ad hoc public that emerged within $24 \mathrm{hr}$ and transformed into a public discourse. Rather than considering the entire population that used the hashtag as a public, Maireder and Schlögl identify multiple publics involved with the topic. Bruns and Burgess (2011) and Bruns and Moe (2014) conceptualize Twitter hashtags as ad hoc publics emerging from within the Twitter community. This conceptualization is based on the view that hashtags act as discursive communities organized around a shared interest. Bruns and Burgess claim that Twitter issue publics can correspond to related issue publics in other public spheres.

In general, online issue publics have well-defined boundaries compared to the broad masses of Internet users identified as public spheres or publics. Previous work highlights that issue publics can be characterized by groups that are well informed and knowledgeable about issues that they perceive to be important and less knowledgeable about issues that are unimportant (Brenes Peralta, Wojcieszak, Lelkes, \& de Vreese, 2017). Members of issue publics may not have mastery over a range of issues, but they are specialists who are "passionately concerned with particular issues on the basis of their values, identities, and interests" (Kim, 2009, p. 255). According to Krosnick (1990), issue publics are driven by personal issue importance, and the attitudes of members of issue publics are linked to other attitudes and resistant to change. Kim (2009) also noted that issue publics in the information environment develop patterns of information acquisition and enhance domain-specific knowledge that results in changes in their engagement in the issue. While issue publics can permeate social media platforms, online collective phenomena should not be restricted to the offline limits of issue publics. For instance, a group of social media users that uses a viral hashtag may not necessarily fall into this category of issue publics, as hashtag networks are broad and are formed in response to the issue rather than expertise, domain-specific knowledge, or special interest. Yet, issue public groups may exist as subsets within the population that used the hashtag. Moreover, as mentioned previously, platform-centric engagement may add "noise" to those subsets by adding large numbers of users that are only momentarily connected to an issue.

\section{Communities}

The notion of communities has been used to explain relatively smaller groups of social media users. For instance, Rosen, Lafontaine, and Hendrickson (2011) see crouchsurfing.com as an online community where members coordinate activity and gather via social media. Similarly, Velasquez (2012) identifies www.lasillavacia.com as an online community characterized by the presence of user profiles, information on the number of posts (comments and stories), points systems based on site contribution, short bios, and private messaging. Stommel and Koole (2010) view an online support group on eating disorders as a community that has normative requirements, which new members are expected to subscribe to. They identify a clear distinction between two related parties (the forum and the newcomer) and describe how the forum acts as a group when establishing interactions with the new member. Albrechtslund's (2010) work on online communities is centered around a World of Warcraft guild, where narratives play a central role in creating a sense of community and collective identity. According to these studies, online communities display more cohesion, interaction among members, and collective identity than publics.

In addition to work that depends on offline metaphors of community to examine online phenomena, current social media research includes a significant number of studies that connect offline behavior with online activity (e.g., Conroy, Feezell, \& Guerrero, 2012; Dunbar, Arnaboldi, Conti, \& Passarella, 2015; Wojcieszak, 2009). In particular, Dunbar et al. (2015) argue that the structure of online communities is similar to offline (face-to-face) networks.

Application of the notion of communities in online settings is as problematic as the idea of online public spheres and publics. While this concept may be appropriate to discuss various online groups examined by some of the studies mentioned above (Rosen et al., 2011; Stommel \& Koole, 2010; Velasquez, 2012) in which users work together in specific settings, this concept may not be appropriate in other contexts. Ren, Kraut, and Kiesler's (2007) observation that communities evolve as members influence the structure of and interaction in online platforms may not be valid in some 
contexts. For instance, those who respond instantly to social/ political issues or natural disasters via means of communication enabled by social media affordances, such as hashtags, may not interact long enough for community features to evolve. Furthermore, several scholars identify the need for new approaches to understanding online spaces. Yuan (2012), for instance, argues that application of online communities does not adequately conceptualize culture. He also suggests a conceptual departure, noting that media-enabled communities may serve as a starting point for examining nuances in technology and social change. Fernback (2007) claims that the "community metaphor placed on virtual social relations is inadequate and inappropriate" (p. 62). This is due to the fact that this metaphor describes only a few qualities of community (fellowship, respect, and tolerance). Fernback also suggests a conceptual departure from the concept and notes:

rather than asking whether or not cyber community is or isn't real community, a long-term perspective on the cultural significance of cyber community focuses on how some users of online technology have created meaningful constructs of social interaction in the online arena. (p. 63)

\section{Theorising Digital Publicness}

We have argued that social media research needs more work examining how platform affordances enable unique aspects of interaction and expression. In this section, we review several concepts that are foundational to such work and build on them to propose a theoretical perspective that captures certain digitally native phenomena.

\section{Affordances}

Affordances, as conceived of in an ecological theory of perception (Gibson, 1979) and subsequently adopted in other fields, are properties of the environment that enable or offer potential for action by an agent. It is important to understand that affordances are not merely properties of the environment: they exist only as a complementary relationship between an agent and its environment (Wells, 2002). Bucher and Helmond (2018) describe how the concept of affordances has been examined from different perspectives, such as high-level and low-level affordances (boyd, 2011), imagined affordances (Nagy \& Neff, 2015), and vernacular affordances (Mcveigh-Schultz \& Baym, 2015). Bucher and Helmond (2018) suggest that platforms may afford different actions for various user types (e.g., end-users, developers), and platform changes (e.g., Twitter "Like" button) may offer possibilities for different actions. We suggest that Twitter hashtags can be seen as affordances for two reasons: (1) the platform affords the creation of hashtags and (2) different actions emerge from the use of hashtags (e.g., discussion, expression, measurement of engagement, and observation of activity).
While concepts of public sphere, publics, and communities help examine dynamics of online activity, they may not fully capture nuances in online activity. This happens for at least two reasons. First, online and offline spaces offer different communicative affordances (e.g., the "grounding constraints" suggested by Clark \& Brennan, 1991) and are ruled by different logics; some rules valid for offline activity may not make much sense online. Framing studies of online contexts within frames developed for offline phenomena may limit the potential for new conceptualizations. Second, contemporary social media function as platforms for different types of activity, and different behavioral practices can emerge on the same platform. For instance, hashtags are a unique aspect within social media that take Twitter users beyond their follower/friendship networks to a more global level of connectivity (Bruns \& Moe, 2014). Explaining this instant connectivity using offline phenomena may be difficult.

\section{Digitally Native}

While questions of whether or not online collectives constitute online public spheres or communities are crucial for our understanding of the networked society, it is important to look beyond these concepts and find "natively digital" ways of conceptualizing online activity. Richard Rogers (2009) highlights the ontological distinction between elements that are "natively digital" and "digitized." The difference between these two relate to the medium in which objects, content, devices, and environments are "born." Rogers (2010) identifies elements that are native to digital media, such as links and tags, as opposed to those that migrated to the Internet via digitization. The scholars who discuss this notion (Marres, 2012; Marres \& Gerlitz, 2016; Niederer \& van Dijck, 2010) pay primary attention to natively digital methods, such as automated collection and analysis of online data. The scope of the natively digital perspective, however, should not be limited to the analysis of digital objects. We suggest that new affordances can enable natively digital social phenomena, such as patterns of interaction and expression that are born in the medium (i.e., the Internet) and can be different from offline communication.

\section{Constructing Publicness}

Holmes (2002) discusses the notion of "public sphericules"- spheres of assimilation with their own dynamics - a concept originally suggested by Todd Gitlin, as an alternative to the conventional public sphere. Holmes notes that individuals using modern communication platforms are in a process of constructing publicness, rather than participating in a given public sphere. This view of fragmented partial publics seems more appropriate to describe the dynamics of online communication. It is also consistent with Latour's (2005) dictum: "no groups, only group formation" (p. 27). 
Rather than argue about what constitutes a public, we should examine the ongoing activity by which participants construct and sustain "publicness" (see also the Fernback quote, previous section). Social phenomena are not fixed entities, but rather are constantly being constructed by participants' actions. However, though fragmented and contingent, people engaging in online activities may perceive being in a larger collective. Marino (2015) discusses "digital togetherness," a sense of belonging and identity based on sharing personal and private experiences (e.g., being online, being native of a particular country, speaking a common language, and having a common ground like being an immigrant). As Rathnayake and Suthers (2016) found, those who are using a hashtag may be locally active, having their interactions limited to a small cluster, but may be globally connected in their imagined affiliation with others through the hashtag. This global-local coexistence needs more "socio-technically established" inquiry.

\section{Phenomenological Aspects}

Our approach to conceptualize the online collective space is based on the premise that social media affordances, such as hashtags, enable creation of quasi-public spaces characterized by both transactive and non-transactive aspects of communication. Uptake of transactive utterances can enable polymorphic user clusters. Although utterances that are non-transactive and are not uptaken do not form clusters that indicate transactions among users, they still provide a sense of being connected to a certain topic. Moreover, although some transactive utterances may not trigger uptake, they still provide a sense of connectedness to the sender. This conception acknowledges that online communication, social media posts in particular, includes phenomenological elements, such as non-other directed expressions that may or may not result in direct conversational transactions among users. This is enabled by the fact that co-presence is not a necessary condition for online communication. The suggested approach provides a holistic view, combining the interactional perspective as well as the work of those who examine psychological aspects of social media activity. Licoppe and Smoreda (2005) argue that the new communication technologies allow creating a new form of sociality, "connected presence," which does not require physical co-presence. Connected presence can be characterized by quasi-continuous exchanges that help people to maintain relationships. We argue that social media can also create an "extended sociality" that includes not only quasicontinuous exchanges but also non other-directed expressions that are driven by a sense of being connected rather than having a dialogue. This state of connectedness is enabled by the ability to interact with platforms rather than a user's follower/friendship network. Hence, maintenance of relationships and engaging discussions are only subsets of online transactions.
Social media literature includes a range of studies that show the phenomenological aspect of mediated communication. For instance, Papacharissi's (2016) notion of affective publics holds that publics are "mobilized and connected (or disconnected) through expressions of sentiment, as these expressions of sentiment materialize discursively through the medium of Twitter" (p. 320). These feelings are supported and sustained by the properties of spreadable technologies. The notion of connectedness we suggest is based on Papacharissi's argument that new media afford structures of connectivity that enable users to engage on an emotional basis. However, not every utterance in these structures, as we argued above, is necessarily discursive. For instance, hashtags may include a large number of tweets that are not directed at others and do not take part in a given discourse. Moreover, hashtag networks can be too polymophic to form a unified discourse as their use spreads beyond follower networks while transactions among those who use hashtags may subject to local level constraints, such as follower networks.

The primacy of the phenomenological aspect in some social media expressions can be supported further through imagined audiences (Litt, 2012) and imagined communities (Gruzd et al., 2011), a mental conceptualization of the audience, rather than an actual audience, that can shape a user's communication via social media. Marwick and boyd's (2011) work on imagined audiences of Twitter users show that while some users have strategic audiences, such as fan bases, some users tend to use Twitter as a diary or a record of their lives; suggesting that public rejection of audience exists within Twitter use. Moreover, Litt and Hargittai's (2016) work show that more than $50 \%$ of users do not think about specific audiences as they share information on social network sites. Litt and Hargittai identify this as "abstract audiences." The above discussion points to the need for concepts that can describe the highly fluid nature of Twitter communication. The ubiquity of abstract audiences (Schmidt, 2014) and Marwick and boyd's (2011) observation of public rejection of audience indicates in particular that there should be a concept that describes an action in a collective context (i.e., tweeting with a hashtag) that does not necessarily have a transactive (otherdirected) communicative intent, though it may have the possibility of responses from others, such as retweets.

\section{MC}

We now examine how publicness is constructed as instances of $\mathrm{MC}$ via the broader variety of interactive relationships that are afforded by Twitter issue-response hashtags. We define momentary connectedness as an interactional and phenomenological computer-mediated publicness that allows polymorphism across layers of communication, such as hashtags, follower networks, and mention/reply relationships (Bruns \& Moe, 2014). MC is an overarching conception that acknowledges the fact that online platforms allow a range of engagement options that are not limited to the boundaries of 


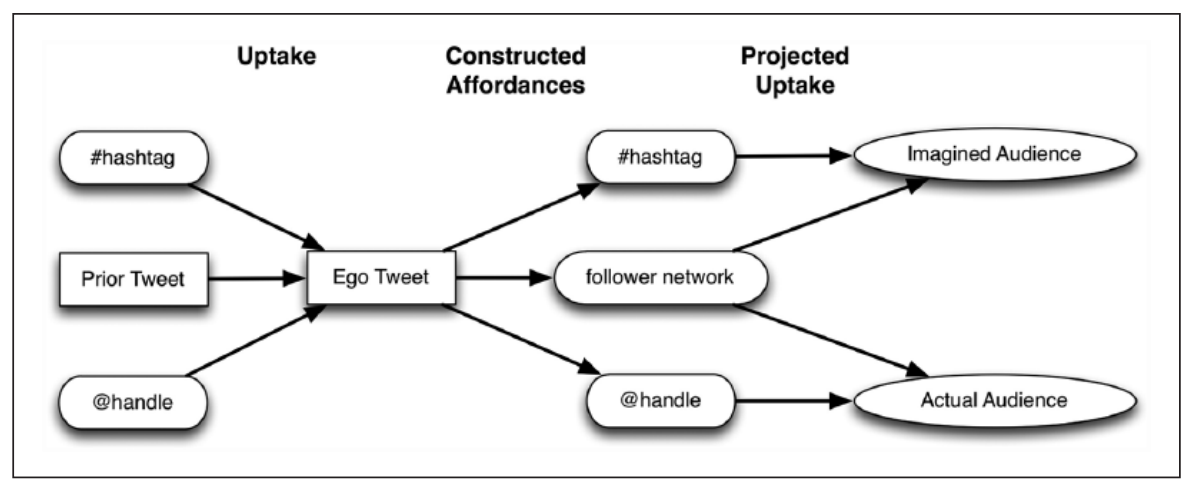

Figure I. Schematic summary.

offline metaphors, and notions such as connected presence, communities, and issue-publics can be seen as manifestations of polymorphism within MC. Sending tweets with an issue-response hashtag is a momentary act that brings users to a topical structure of connectivity. That, however, is not necessarily an indication of user intention to form or interact with well-defined, and unified, communities or publics. This structure of connectivity is a combination of non-other directed expressions and mere repetition of messages, as well as clusters of active users engaged in the issue. The term "momentary connectedness" captures the emergence of these structures of connectivity in hashtag networks as constructed by uptake organized around a hashtag. Hashtag networks as instances of MC are enabled by the affordances of the platform. The \# character followed by a topic name, as interpreted by Twitter's technological infrastructure, functions as a tool for mobilizing and focusing collective activity. It does so by serving as a prompt, inviting response on the indicated topic. The hashtag brings the contributions of those who take up this invitation into a mutually visible space. In these ways, a hashtag functions as an "immutable mobile" (Latour, 1986). The affordances enable different layers of mediated communication, such as tweets not targeted at any user, as well as retweet, reply, and mention clusters, and acts of liking and private messages.

\section{Uptake and Projected Uptake}

Scholarly work along several dimensions is necessary to situate the theoretical construct of MC in empirical settings. In the following discussion, we take a generalized relational perspective to substantiate MC. We use the notion of "uptake" to situate our conception of interaction between participants in a more natively digital context. Uptake is the most fundamental element of collective action. Originally conceived of as "acts in which one participant takes up another's contribution and does something further with it" (Suthers, 2006b, p. 331 ), the concept was generalized in order to encompass a broader and media-independent conception of interactional relationships, and defined more formally as "the relationship present when a participant's coordination takes aspects of prior or ongoing events as having relevance for an ongoing activity" (Suthers et al., 2010, p. 9). Uptake is a broader phenomenon than "transactivity," an element of interaction that Stahl (2013) defines as "the reasoning of one utterance building on another utterance's reasoning" (p. 1). While the focus of transactivity is limited to contributions explicitly directed at others' contributions, uptake allows analysis of situations where interactions can occur without the necessity of either actor intending to address the other. Also, uptake abstracts from media-specific relationships such as "reply to post" or "adjacency pair," and thus can capture participatory structures within multiple media or even across media (Suthers, 2006a). This concept is appropriate to discuss the fluid nature of online communication. Uptake helps understand how individual actions aggregate to larger entities, and it can be applied across platforms and different affordances.

While uptake is useful for conceptualizing how explicitly interactive online expressions such as retweets and replies build on past activity, there is a need for a concept that can capture how acts are projected to future activity. We suggest that this phenomenological element can be brought into the interactional domain of investigation by recognizing the projected uptake of online expressions. When one constructs a media expression that contains affordances for action (constructed affordances), then projected uptake is the potential that others will take up these affordances. The choice of constructed affordance indicates the kind of uptake that is projected (Figure 1). For instance, a tweet that is not directed at specific users may trigger retweets and replies in the future, as Twitter affordances allow such uptake. Although uptake is explicit, projected uptake may not be directed at explicit audiences. Accordingly, projected uptake captures the possibilities of abstract audiences (Litt \& Hargittai, 2016) and public rejection of audience (Marwick \& boyd, 2011).

Networked instances of MC can be constructed from a variety of other-directed and non-directed forms of uptake and projected uptake. Accordingly, MC shows the co-existence of concrete collective phenomena, such as interactions among individuals in issue publics or communities, as well 
as individual users who do not display any discursive intent. This, we argue, characterizes an expanded sociality enabled by social media affordances. Figure 1 shows a schematic summary of the interplay between uptake, social media affordances, and projected uptake. In issue-response contexts, tweets (ego tweets in Figure 1) can result from the uptake of a hashtag (i.e., original tweets that contain the hashtag), prior tweets (i.e., retweets), as well as the "@handle" (i.e., ‘@replies). These tweets can be projected to an abstract population of individuals that may or may not use the hashtag, an imagined group of individuals in a users' follower network, as well as actual users specified by the use of the "@handle."

\section{MC in IRNs}

This article is primarily theoretical. However, to illustrate empirical relevance, we present evidence of two aspects of MC: (1) uptake and projected uptake in Twitter IRNs and (2) the existence of polymorphic structures. This analysis helps establish the notion of $\mathrm{MC}$ as it shows how non-other directed and primarily phenomenological expressions as well as user clusters with different orientations co-exist in hashtag networks. Accordingly, the objective of the following empirical analysis is to establish, using Twitter activity related to the April 2015 earthquake in Nepal as an empirical context, the existence of and examine polymorphism in IRNs, including (1) the co-presence of transactive, non-transactive, and projected uptake and (2) structural polymorphism of collective activity.

\section{Method}

We use a Twitter hashtag network dataset representing \#NepalEarthquake, a viral hashtag used in response to the April 2015 earthquake in Nepal, to achieve the above objective. This dataset was collected during the first week after the earthquake using the Twitter API plugin in the NodeXL template. The Twitter Search API returns a collection of tweets that match a given query (\#NepalEarthquake in this case) at a specific time. The earthquake occurred on 25 April, and our data included a sample of tweets (including original tweets, retweets, and replies) covering a period of 2 days after the disaster. The sample is neither random nor complete. However, the API provided a significantly large collection of Tweets to establish the existence of mixed transactive intent and structural polymorphism of collective activity. The 15,118 nodes in the dataset represent users who tweeted, retweeted, or replied with the hashtag. Edges that represent retweets and @replies form connectivity in the network (16,107 edges in our data). For instance, a retweet creates an edge from the sender to the user whose message was retweeted. Similarly, a reply creates an edge from the sender to the person who is replied to in the tweet. Original tweets have self-loops, the third type of edge, which are removed from our network analysis. The dataset included 1898 original tweets. The Nepal earthquake constitutes a suitable case to examine IRNs for several reasons. First, a natural disaster of high magnitude can motivate instant social media activity. Second, the vast popularity of the Himalayan range as a top tourist destination can trigger activity beyond geographic or national boundaries. Third, a natural disaster may trigger a range of activities that focus on actual disaster relief support as well as mere emotional engagement (e.g., "prayer tweets") that allows examining phenomenological elements of social media activity.

\section{Uptake and Projected Uptake in \#NepalEarthquake}

Table 1 shows examples of uptake and projected uptake in the \#NepalEarthquake tweets. These examples show that the space defined by the hashtag includes messages that do not display explicit evidence of interactional intent, quasi-interactional exchanges that begin to form collective activity, as well as messages that are explicitly transactive to a primary audience. Lack of clear projection for future uptake and imagined or abstract audiences are connected to tweeting at each level. This shows that hashtag IRNs are complex structures with varying levels of interaction.

From an interactional perspective, by not mentioning other users in a space where that is possible (and is a common practice), original tweets (those that are not retweets, quote tweets, mentions, or replies) indicate less interactive intent than retweets or @ replies. This is particularly the case when users do not imagine audiences or imagine broad audiences beyond their reach. However, a tweet has interactional intent when it is projected into the future expecting future uptake. From a strict relational viewpoint, original tweets do not contribute to a conversational structure until other users in the network take up the message (for retweeting or quote tweeting). Therefore, compared to replies, mentions, retweets, and quote tweets, original tweets do not directly construct collective action. It is possible that users who send tweets with a hashtag imagine specific audiences, broad audiences, or even real online communities. On the other hand, it is also possible that users do not imagine audiences at all as tweeting may be a habitual action or tweeting itself may provide a gratification for the user. For instance, one of the subjects of Marwick and boyd's (2010, p.119) study mentioned that tweeting is not directed toward anyone, but rather he or she does it "to do it." This, however, does not mean that those utterances happen in a non-communicative space. As Marwick and boyd note, those individuals do not tweet to a void as they are bound by follower-followee relationships.

A reasonable number of original tweets in the \#NepalEarthquake were either prayers or thoughts in the form of tweets. The collection of tweets included the word pray in some form (e.g., prayers, praying, sendingprayers) 2012 times. Arguably, unless organized as a public event (e.g., candle vigil 
Table I. Uptake and Projected Uptake in \#NepalEarthquake Tweets.

\begin{tabular}{|c|c|c|c|c|}
\hline Message type & Affordance & Examples from \#NepalEarthquake Data & Uptake & Projected uptake \\
\hline Original Tweet & Hashtag & $\begin{array}{l}\text { Really difficult day yesterday . . . in addition } \\
\text { to aftershocks . . . rainfall . . . too much } \\
\text { hardship \#NepalEarthquake }\end{array}$ & Hashtag & $\begin{array}{l}\text { Imagined, abstract } \\
\text { audiences, or may not } \\
\text { intend future uptake }\end{array}$ \\
\hline Original Tweet & Hashtag & $\begin{array}{l}\text { Let's pray for the victims and their } \\
\text { families in Nepal .... \#NepalEarthquake } \\
\text { \#PrayforNepal [URL] }\end{array}$ & Hashtag & $\begin{array}{l}\text { Imagined or abstract } \\
\text { audiences }\end{array}$ \\
\hline Retweet & Retweet Function & $\begin{array}{l}\text { RT @unicefusa: IM children require } \\
\text { urgent humanitarian assistance after } \\
\text { \#NepalEarthquake. Help now-donate to } \\
\text { UNICEF: [URL] }\end{array}$ & $\begin{array}{l}\text { Prior Tweet/ } \\
\text { Sender }\end{array}$ & $\begin{array}{l}\text { Imagined or abstract } \\
\text { audiences }\end{array}$ \\
\hline $\begin{array}{l}\text { Retweet with a } \\
\text { mentioned @handle }\end{array}$ & $\begin{array}{l}\text { Retweet function } \\
\text { and the @handle }\end{array}$ & $\begin{array}{l}\text { RT @eoiktmnp: Buses from Pokhra to } \\
\text { Raxaul and Kathmandu to Gorakhpur will } \\
\text { also evacuate Indian citizens @meaindia } \\
\text { \#nepalearthquake }\end{array}$ & $\begin{array}{l}\text { Prior Tweet/ } \\
\text { Sender }\end{array}$ & $\begin{array}{l}\text { Specific primary } \\
\text { audience and imagined } \\
\text { secondary audiences }\end{array}$ \\
\hline Reply & @handle & $\begin{array}{l}\text { @wailers, we're raising funds for } \\
\text { \#NepalEarthquake [URL]; would love your } \\
\text { support in spreading the word! }\end{array}$ & $\begin{array}{l}\text { Prior/External } \\
\text { Actor }\end{array}$ & $\begin{array}{l}\text { Specific primary } \\
\text { audience and imagined } \\
\text { secondary audiences }\end{array}$ \\
\hline
\end{tabular}

or a group prayer), prayers are private, or at least non-transactive activities. "Prayer tweets" do not require imagining audiences. However, Twitter hashtags still shift the sender from a private to a more connected state. The dataset included tweets that were directed at those who were affected by the disaster, rather than another Twitter user (e.g., "I hope everyone from \#Nepal is ok This is the pray for you from \#Myanmar \#NepalEarthquake \#instagram"). Arguably, it is possible that the primary function of these tweets is to express thoughts rather than talking to a follower network. The dataset also included tweets that showed some directedness, but display lack of identification of a specific audience (e.g., "Lets PRAY for the victims. \#NepalEarthquake"). The absence of a direct target receiver for these messages situates them in the realm of messages aimed at imagined audiences. Lack of explicit interaction via mentioning names, spreading messages (via retweeting), or replying, as well as the blurry nature of audience in original tweets make it difficult to put the tweets in strict conceptual categories, such as issue publics or online communities. Therefore, original tweets (excluding retweets and @replies) should be situated in a broad conceptual category that does not necessarily include an actual audience. We argue that such messages can be seen as indicative of a state of connectedness where users shift from communication within their follower networks to a topical structure of connectivity. This view is consistent with the claim that Twitter imagined communities are both collective and personal at once (Gruzd et al., 2011) as users make these non-otherdirected utterances in a space defined by a common topic (the hashtag) and a population of users using the platform in response to the disaster.

\section{Polymorphism}

Rathnayake and Suthers (2016) observed that \#illridewithyou, a hashtag that emerged in response to the Lindt
Chocolate Café shooting incident that occurred in Sydney in December 2014, is a highly clustered network in which actors interacted more frequently within each cluster than with the rest of the network. Clustering methods can help show the extent of polymorphism in hashtag networks. In this section, we examine snapshots of Twitter clusters within the \#NepalEarthquake network to illustrate the variety of structures within MC.

Within social network analysis, "community detection" refers to methods of finding cohesive subgraphs of networks (Fortunato, 2010), not to be confused with sociological concepts of community. These algorithms can uncover more broadly conceived and even unanticipated forms of connectivity emerging in online environments, including hashtagenabled user clusters. This is an important capability, as new media affordances enable collective behaviors that may display properties different from those of offline publics or communities. Algorithmic community detection is an active area of research, and dozens of algorithms are available (e.g., Fortunato, 2010). Modularity, a measure of the extent to which a partitioning of a network identifies clusters that have strong internal connectivity (Newman, 2006), forms the basis for several algorithms. One such algorithm is the Louvain method (Blondel, Guillaume, Lambiotte, \& Lefebvre, 2008). The Louvain method performs well on benchmarks (Barabási, 2016) and can identify clusters that are interpretable in terms of social activity (Suthers, 2017; Suthers, Fusco, Schank, Chu, \& Schlager, 2013). Another strong contender, InfoMap (Rosvall, Axelsson, \& Bergstrom, 2009) is an information theoretic approach based on the metaphor of defining the most compact "map" to describe an ergodic random walk in the graph. Due to Louvain's resolution limit and InfoMap's horizon limit, InfoMap produces a finer grained partition with our data, but the partitions are similar (the normalized mutual information between the two 
Table 2. Polymorphic Clusters in the \#NepalEarthquake Network.

\begin{tabular}{ll}
\hline Partition & Characteristics \\
\hline $\begin{array}{l}\text { (a) One Direction fan } \\
\text { cluster ( } 947 \text { nodes }\end{array}$ & $\begin{array}{l}\text { Fans who retweeted a message sent by the music band. Fans themselves are not connected. The } \\
\text { main function of clusters of this nature is to uptake a powerful message and spread it among } \\
\text { follower networks }\end{array}$ \\
$\begin{array}{l}\text { (b) Non-profit support } \\
\text { Indicates some concentration of interaction: } 9 \text { of the top I5 nodes (by degree) were non-profit } \\
\text { and } 873 \text { edges) }\end{array}$ & $\begin{array}{l}\text { organizations (e.g., World Vision Australia, Australian Red Cross, UNICEF). May resemble an offline } \\
\text { issue public within which clusters of users have become momentarily connected online around the }\end{array}$ \\
\#NepalEarthquake topic \\
(c) New cluster (505 & Central nodes are news organizations or news-related nodes, such as The Hindu, Indian Express, \\
nodes and 690 edges) & and Times Now
\end{tabular}

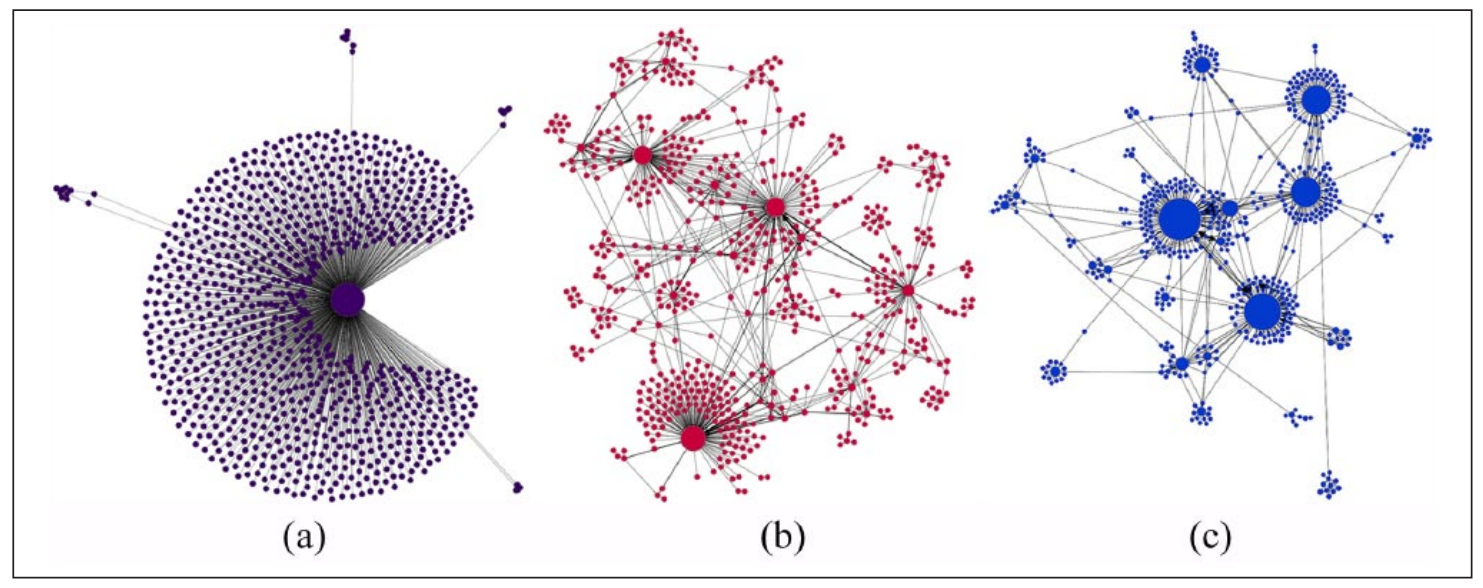

Figure 2. Polymorphic Clusters in the \#NepalEarthquake Network: (a) One Direction fan cluster, (b) non-profit support cluster, and (c) news cluster.

methods on our data is 0.88 ). We prefer the coarser partition because it shows larger connected structures, but the conclusions of this section are not affected.

We partitioned our \#NepalEarthquake network using Gephi's (gephi.org) implementation of the Louvain method. Table 2 provides details on three partitions to illustrate diverse forms of momentary connectivity within the \#NepalEarthquake network (Figure 2). These three partitions consist of different user groups and display different orientations. While the first partition is a subset of a Twitter fan network that indicate 'slacktivism,' the second partition is organized based on special interest, topical relevance, and expertise, which can (loosely) meet criteria for issue publics. The third cluster is primarily driven by the motive to take up breaking news and share with users' followers.

Although the above clusters are different from each other, they should not be understood as a collection of disjoint spaces since $78.6 \%$ of the 15,118 nodes (including these clusters) are in the largest weakly connected component. This indicates that above partitions exist as polymorphic clusters in a broader space of connectivity. While these clusters display properties of offline collective activity to a certain extent, they are only subsets of a larger space that we conceptualize in this study. Although we can find elements of interactivity that reflect some existing models in IRNs, the network itself is a broader phenomenon, an interactional and phenomenological computer-mediated publicness that is polymorphic across layers of communication. Accordingly, $\mathrm{MC}$ does not reject other novel conceptualizations, such as connected presence (Licoppe \& Smoreda, 2005; Miller, 2008), online public sphericules (Holmes, 2002), and digital togetherness (Marino, 2015) as well as online manifestations of offline phenomena, such as issue publics and communities. Rather, it serves as an overarching conceptualization, an expanded sociality, that allows such phenomena.

\section{Conclusion}

This work responds to calls for new conceptualizations of online activity, such as how users have created useful constructs of social interaction online (Fernback, 2007) and the ways in which we construct publicness online (Holmes, 2002). Conceptualizing contemporary social media is difficult as these platforms facilitate various types of communicative actions that are appropriated by users in different ways, rather than being tools that serve specific purposes. New forms of engagement emerging from the interaction between platform design and users demand socio-technical readings of 
online activity. This article situates the act of tweeting in a relational context, providing a window to understand original tweets in comparison to @replies and retweets. Accordingly, we suggest that Twitter issue-response hashtags can be seen as affordances for MC enabled by how the platform handles visibility. Tweets that display broad and vague audience dimensions, as well as clusters with very different orientations within the same topical network, show that hashtags are affordances for MC in which different communicative phenomena can co-exist. Users may potentially navigate between real and imagined audiences, and hashtags both afford actual (enacted) uptake and projection for potential future uptake. $\mathrm{MC}$ can have a phenomenological meaning based on both the user's experience of feeling connected and the network of associations constructed from both explicit uptake and projected uptake that merit having this experience. $\mathrm{MC}$ is in part a structural claim about connectedness. At the same time, it is a claim about the psychological state of those who tweet. In other words, the structure is in part phenomenological.

From an overall point of view, this article provides a novel perspective to understand hashtagged exchanges that does not depend on offline metaphors. We argue that MC constitutes a "natively digital" collective phenomenon. This, as we argued above, does not mean that MC is completely separate from models that resemble offline phenomena. Although new media platforms give rise to natively digital objects as Rogers (2010) argued, we argue that natively digital social phenomena do not completely exclude offline elements, as online interactions occur in a broader offline social context. In other words, it is highly unlikely that computer networks enable purely native online social phenomena.

$\mathrm{MC}$ situates the act of tweeting in a broad domain that can be public as well as quasi-public. We understand their acts using the analytic concept of "uptake" (Suthers et al., 2010) - the fundamental relationship between actions out of which interaction (and ultimately social ties) is constructedbecause it is independent of media and assumptions of transactivity (Suthers, 2006b). This enables us to develop conceptions of technologically embedded social phenomena that are not derived from offline analogs. Uptake encompasses transactive relationships (e.g., replies), nontransactive relationships (e.g., retweets), and the potential for future relationships when a tweet is projected into an issue-topic hashtag space. Participants enact these various forms of uptake and projected uptake through the affordances of hashtags to construct networks of MC that meet their needs for expressing responses to catastrophes and other events in the context of actual and imagined collectives. It is important to note that the notions of uptake and projected uptake are not limited to the notion of MC. While uptake is the most fundamental element of actual interaction, projected uptake captures the potential forms of interaction, particularly in networked media. Therefore, these two elements can be applied to study actual and potential interaction in different online platforms.
As argued before, online engagement can be platform-centric as the users are exposed to a range of topics at the same time. Simultaneous exposure to a range of issues may minimize engagement to effortless acts (e.g., liking, retweeting) unless users (and/or groups of users) have a special interest in a given issue. Therefore, slacktivism as well as organized collective effort co-exist among groups of people that are bound together by a common characteristic. This can happen on multiple levels. The example discussed above shows how this occurs on the level of hashtags. Further research may examine how users are momentarily connected to multiple issues on the platform level. For instance, a user may marginally engage with a particular issue while leading an active conversation related to another issue. Further work is necessary to examine how users navigate between issues and engage with them in different degrees.

The contribution of this study is primarily theoretical. Therefore, the empirical analysis of the \#NepalEarthquake sample should not be considered exhaustive. While network analysis can help demonstrate polymorphism, more work is necessary to examine temporal changes in user clusters. Moreover, qualitative methods such as interviews can help understand the phenomenological aspect of MC. Although discussed in the context of disaster response hashtags, the notion of MC can be applied to other types of issue-response hashtags. Polymorphism and the extent of non-transactive expressions may depend on the nature of the hashtag. Further work is necessary to examine the compatibility of this notion with other types of hashtag networks. Moreover, future work may focus on $\mathrm{MC}$ in different platforms. Future studies may also examine how multiple communities and/or publics are engaged in MC. From an individual user's perspective, it is important to examine the simultaneous participation in a range of momentary discourses, issues, or events. More broadly, other social media also provide affordances for MC and await further investigation. Further work may also focus on substantiating the claim that subsets of users engage in actions that may reflect offline phenomena within MC.

\section{Declaration of Conflicting Interests}

The author(s) declared no potential conflicts of interest with respect to the research, authorship, and/or publication of this article.

\section{Funding}

The author(s) received no financial support for the research, authorship, and/or publication of this article.

\section{ORCID iD}

Chamil Rathnayake (D) https://orcid.org/0000-0003-1964-2639

\section{References}

Al-Ani, B., Mark, G., Chung, J., \& Jones, J. (2012). The Egyptian blogosphere: A counter-narrative of the revolution. In Proceedings of the ACM 2012 Conference on Computer 
Supported Cooperative Work (pp. 17-26). Retrieved from https://dl.acm.org/citation.cfm?id=2145204\&picked=prox

Albrechtslund, A. (2010). Gamers telling stories: Understanding narrative practices in an online community. Convergence: The International Journal of Research into New Media Technologies, 16, 112-124. doi:10.1177/1354856509348773

Ausserhofer, J., \& Maireder, A. (2013). National politics on Twitter: Structures and topics of a networked public sphere. Information, Communication \& Society, 16, 291-314. doi:10.1 080/1369118X.2012.756050

Barabási, A.-L. (2016). Network science. Cambridge, UK: Cambridge University Press.

Blondel, V. D., Guillaume, J.-L., Lambiotte, R., \& Lefebvre, E. (2008). Fast unfolding of communities in large networks. Journal of Statistical Mechanics: Theory and Experiment, 2008, P10008.

Boeder, P. (2005). Habermas' heritage: The future of the public sphere in the network society. First Monday, 10(9), 1-10.

boyd, d. (2011). Social Network Sites as networked publics: Affordances, dynamics, and implications. In Z. Papacharissi (Ed.), A networked self: Identity, community, and culture on social network sites (pp. 39-58). New York, NY: Routledge Taylor \& Francis Group. doi:10.4324/9780203876527

Brenes Peralta, C., Wojcieszak, M., Lelkes, Y., \& de Vreese, C. (2017). Selective exposure to balanced content and evidence type: The case of issue and non-issue publics about climate change and health care. Journalism \& Mass Communication Quarterly, 94, 833-861. doi:10.1177/1077699016654681

Brock, A. (2012). From the blackhand side: Twitter as a cultural conversation. Journal of Broadcasting \& Electronic Media, 56, 529-549.

Bruns, A., \& Burgess, J. (2011). The use of Twitter hashtags in the formation of ad hoc publics. Paper presented at the 6th European Consortium for Political Research General Conference, 25-27 August, University of Iceland, Reykjavik, Iceland.

Bruns, A., Burgess, J., Highfield, T., Kirchhoff, L., \& Nicolai, T. (2011). Mapping the Australian networked public sphere. Social Science Computer Review, 29, 277-287. doi:10.1177/ 0894439310382507

Bruns, A., \& Moe, H. (2014). Structural layers of communication on Twitter. In K. Weller, A. Bruns, J. Burgess, M. Mahrt, \& C. Puschmann (Eds.), Twitter and society (pp. 15-28). New York, NY: Peter Lang.

Bruns, A., Moon, B., Paul, A., \& Münch, F. (2016). Towards a typology of hashtag publics: A large-scale comparative study of user engagement across trending topics. Communication Research and Practice, 2, 20-46. doi:10.1080/22041451.2016.1155328

Bucher, T., \& Helmond, A. (2018). The affordances of social media platforms. In J. Burgess, A. Marwick, \& T. Poell (Ed.), The SAGE handbook of social media (pp. 233-253). London, England: SAGE.

Clark, H. H., \& Brennan, S. E. (1991). Grounding in communication. In L. B. Resnick, J. M. Levine, \& S. D. Teasley (Eds.), Perspectives on socially shared cognition (pp. 127-149). Washington, DC: American Psychological Association.

Conroy, M., Feezell, J. T., \& Guerrero, M. (2012). Facebook and political engagement: A study of online political group membership and offline political engagement. Computers in Human Behavior, 28, 1535-1546. doi:10.1016/j. chb.2012.03.012
Dahlgren,P.(2005). TheInternet, publicspheres, and politicalcommunication: Dispersion and deliberation. Political Communication, 22, 147-162. doi:10.1080/10584600590933160

Dunbar, R. I. M., Arnaboldi, V., Conti, M., \& Passarella, A. (2015). The structure of online social networks mirrors those in the offline world. Social Networks, 43, 39-47. doi:10.1016/j.socnet.2015.04.005

El-Nawawy, M., \& Khamis, S. (2010). Collective identity in the virtual Islamic public sphere. International Communication Gazette, 72, 229-250. doi:10.1177/1748048509356949

Evans, S. K., Pearce, K. E., Vitak, J., \& Treem, J. W. (2017). Explicating affordances: A conceptual framework for understanding affordances in communication research. Journal of Computer-Mediated Communication, 22, 35-52. doi:10.1111/ jcc4.12180

Fernback, J. (2007). Beyond the diluted community concept: A symbolic interactionist perspective on online social relations. New Media \& Society, 9, 49-69. doi:10.1177/1461444807072417

Florini, S. (2014). Tweets, tweeps, and signifyin': Communication and cultural performance on "Black Twitter." Television \& New Media , 15, 223-237. doi:10.1177/1527476413480247

Fortunato, S. (2010). Community detection in graphs. Physics Reports, 486, 75-174. doi:10.1016/j.physrep.2009.11.002

Frederick, E., \& Neuwirth, K. (2008). The second-person effect and its role in formation of active issue publics. Mass Communication and Society, 11, 514-538. doi:10.1080/15205430802368613

Geiger, R. S. (2009). Does Habermas understand the internet? The algorithmic construction of the blogo/public sphere. Gnovis: A Journal of Communication, Culture, and Technology, 10(1), 1-29. Retrieved from http://gnovisjournal.org/journal/does-habermasunderstand-internet-algorithmic-construction-blogopublic-sphere

Gibson, J. J. (1979). The theory of affordances. In J. J. Gibson (Ed.), The ecological approach to visual perception (pp. 119-136). Boston, MA: Houghton Mifflin.

Gruzd, A., Wellman, B., \& Takhteyev, Y. (2011). Imagining Twitter as an imagined community. American Behavioral Scientist, 55, 1294-1318. doi:10.1177/0002764211409378

Himelboim, I., Hansen, D., \& Bowser, A. (2013). Playing in the same Twitter network: Political information seeking in the 2010 US gubernatorial elections. Information, Communication \& Society, 16, 1373-1396.

Holmes, D. (2002). Transformations in the mediation of publicness: Communicative interaction in the network society. Journal of Computer-Mediated Communication, 7(2). doi:10.1111/j.1083-6101.2002.tb00142.x

Kim, Y. M. (2009). Issue publics in the new information environment. Communication Research, 36, 254-284. doi:10.1177/ 0093650208330253

Krosnick, J. A. (1990). Government policy and citizen passion: A study of issue publics in contemporary America. Political Behavior, 12, 59-92.

Kruckeberg, D., \& Vujnovic, M. (2010). The death of the concept of publics (plural) in 21st century public relations. International Journal of Strategic Communication, 4, 117125. doi:10.1080/15531181003701921

Latour, B. (1986). Visualisation and cognition: Drawing things together. In E. Long \& H. Kuklick (Eds.), Knowledge and society: Studies in the sociology of culture past and present (Vol. 6, pp. 1-40). New York, NY: Elsevier Science. doi:10.1002/9780470979587.ch9 
Latour, B. (2005). Reassembling the social: An introduction to Actor-Network theory. Oxford, UK: Oxford University Press. doi:10.1163/156913308X336453

Licoppe, C., \& Smoreda, Z. (2005). Are social networks technologically embedded? Social Networks, 27, 317-335. doi:10.1016/j. socnet.2004.11.001

Litt, E. (2012). Knock, knock. Who's there? The imagined audience. Journal of Broadcasting \& Electronic Media, 56, 330345. doi:10.1080/08838151.2012.705195

Litt, E., \& Hargittai, E. (2016). The imagined audience on social network sites. Social Media + Society, 2, 1-12. doi:10.1177/2056305116633482

Maireder, A., \& Schlögl, S. (2014). 24 hours of an \# outcry: The networked publics of a socio-political debate. European Journal of Communication, 29, 687-702. doi:10.1177/0267323114545710

Marino, S. (2015). Making space, making place: Digital togetherness and the redefinition of migrant identities online. Social Media + Society, 1, 1-9. doi:10.1177/2056305115622479

Marres, N. (2012). The redistribution of methods: On intervention in digital social research, broadly conceived. The Sociological Review, 60(Suppl. 1), 139-165. doi:10.1111/j.1467-954X.2012.02121.x

Marres, N., \& Gerlitz, C. (2016). Interface methods: Renegotiating relations between digital social research, STS and sociology. The Sociological Review, 64, 21-46. doi:10.1111/1467954X.12314

Marwick, A. E., \& boyd, d. (2010). I tweet honestly, I tweet passionately: Twitter users, context collapse, and the imagined audience. New Media \& Society, 13, 114-133. doi:10.1177/ 1461444810365313

Mcveigh-Schultz, J., \& Baym, N. K. (2015). Thinking of you: Vernacular affordance in the context of the microsocial relationship app, Couple. Social Media + Society, 1, 1-13. doi:10.1177/2056305115604649

Mejias, U. A. (2010). The limits of networks as models for organizing the social. New Media \& Society, 12, 603-617. doi: $10.1177 / 1461444809341392$

Miller, V. (2008). New media, networking and phatic culture. Convergence: The International Journal of Research into New Media Technologies, 14, 387-400. doi:10.1177/ 1354856508094659

Nagy, P., \& Neff, G. (2015). Imagined affordance: Reconstructing a keyword for communication theory. Social Media + Society, 1, 1-9. doi:10.1177/2056305115603385

Newman, M. E. J. (2006). Modularity and community structure in networks. Proceedings of the National Academy of Sciences of the United States of America, 103, 8577-8582. doi:10.1073/ pnas.0601602103

Niederer, S., \& van Dijck, J. (2010). Wisdom of the crowd or technicity of content? Wikipedia as a sociotechnical system. New Media \& Society, 12, 1368-1387. doi:10.1177/1461444810365297

Papacharissi, Z. (2002). The virtual sphere: The internet as a public sphere. New Media \& Society, 4, 9-27. doi:10.1177/ 14614440222226244

Papacharissi, Z. (2016). Affective publics and structures of storytelling: Sentiment, events and mediality. Information, Communication\&Society, 19,307-324.doi:10.1080/1369118X. 2015.1109697

Rathnayake, C., \& Suthers, D. D. (2016). Networked solidarity: An exploratory network perspective on twitter activity related to \#illridewithyou. In Proceedings of the Annual Hawaii International Conference on System Sciences. (Vol. 2016, pp. 2058-2067, IEEE). doi:10.1109/HICSS.2016.259 Retrieved from https://ieeexplore.ieee.org/document/7427440/

Rauchfleisch, A., \& Kovic, M. (2016). The Internet and generalized functions of the public sphere: Transformative potentials from a comparative perspective. Social Media + Society, 2(2), 1-15. doi:10.1177/2056305116646393

Ren, Y., Kraut, R., \& Kiesler, S. (2007). Applying common identity and bond theory to design of online communities. Organization Studies, 28, 377-408. doi:10.1177/0170840607076007

Rogers, R. (2009). The end of the virtual: Digital methods. Amsterdam, The Netherlands: Vossiuspers UvA.

Rogers, R. (2010). Internet research: The question of methodA keynote address from the YouTube and the 2008 Election Cycle in the United States Conference. Journal of Information Technology \& Politics, 7, 241-260. doi:10.1080/ 19331681003753438

Rosen, D., Lafontaine, P. R., \& Hendrickson, B. (2011). CouchSurfing: Belonging and trust in a globally cooperative online social network. New Media \& Society, 13, 981-998. doi:10.1177/1461444810390341

Rosvall, M., Axelsson, D., \& Bergstrom, C. T. (2009). The map equation. European Physical Journal: Special Topics, 178, 13-23. doi:10.1140/epjst/e2010-01179-1

Schmidt, J. (2014). Twitter and the rise of personal publics. In K. Weller, A. Bruns, J. Burgess, M. Mahrt, \& C. Puschmann (Eds.), Twitter and society (pp. 3-14). New York, NY: Peter Lang.

Shklovski, I., \& Valtysson, B. (2012). Secretly political: Civic engagement in online publics in Kazakhstan. Journal of Broadcasting \& Electronic Media, 56, 417-433.

Stahl, G. (2013). Transactive discourse in CSCL. International Journal of Computer-Supported Collaborative Learning, 8, 145-147. doi:10.1007/s11412-013-9171-6

Stommel, W., \& Koole, T. (2010). The online support group as a community: A micro-analysis of the interaction with a new member. Discourse Studies, 12, 357-378. doi:10.1177/ 1461445609358518

Suthers, D. D. (2006a). A qualitative analysis of collaborative knowledge construction through shared representations. Research and Practice in Technology Enhanced Learning, 1(2), 1-28.

Suthers, D. D. (2006b). Technology affordances for intersubjective meaning making: A research agenda for CSCL. International Journal of Computer-Supported Collaborative Learning, 1, 315-337. doi:10.1007/s11412-006-9660-y

Suthers, D. D. (2017). Applications of cohesive subgraph detection algorithms to analyzing socio-technical networks. In Proceedings of the 50th Hawaii International Conference on System Sciences (pp. 2128-2137). New York, NY: IEEE.

Suthers, D. D., Dwyer, N., Medina, R., \& Vatrapu, R. (2010). A framework for conceptualizing, representing, and analyzing distributed interaction. International Journal of ComputerSupported Collaborative Learning, 5, 5-42. doi:10.1007/ s11412-009-9081-9

Suthers, D. D., Fusco, J., Schank, P., Chu, K. H., \& Schlager, M. (2013). Discovery of community structures in a heterogeneous professional online network. In Proceedings of the Annual 
Hawaii International Conference on System Sciences (pp. 3262-3271, IEEE). doi:10.1109/HICSS.2013.179

Tremayne, M., Zheng, N., Lee, J. K., \& Jeong, J. (2006). Issue publics on the web: Applying network theory to the war blogosphere. Journal of Computer-Mediated Communication, 12, 290-310. doi:10.1111/j.1083-6101.2006.00326.x

Velasquez, A. (2012). Social media and online political discussion: The effect of cues and informational cascades on participation in online political communities. New Media \& Society, 14, 1286-1303. doi:10.1177/1461444812445877

Warner, M. (2002). Publics and counterpublics (abbreviated version). Quarterly Journal of Speech, 88, 413-425.

Wells, A. J. (2002). Gibson's affordances and Turing' s theory of computation. Ecological Psychology, 14, 140-180. doi:10.1207/ S15326969ECO1403

Wojcieszak, M. (2009). "Carrying online participation offline": Mobilization by radical online groups and politically dissimilar offline ties. Journal of Communication, 59, 564-586. doi:10.1111/j.1460-2466.2009.01436.x
Yuan, E. J. (2012). A culturalist critique of "online community" in new media studies. New Media \& Society, 15, 665-679. doi:10.1177/1461444812462847

\section{Author Biographies}

Chamil Rathnayake ( $\mathrm{PhD}$, University of Hawaii) is a lecturer in the School of Humanities at the University of Strathclyde. His current research focuses on social media affordances, issue-response networks, and cross-ideology exposure, with a special emphasis on the ways in which new media affordances restructure collective activity.

Daniel D. Suthers (PhD, University of Massachusetts) is a Professor in the Department of Information and Computer Sciences at the University of Hawaii at Manoa. His research is generally concerned with cognitive, social, and computational perspectives on designing and evaluating software for learning, collaboration, and community, with a current focus on social affordances of digital media and multilevel analysis of sociotechnical systems. 